\title{
Weak Set-Labeling Number of Certain Integer Additive Set-Labeled Graphs
}

\author{
N. K. Sudev \\ Department of Mathematics \\ Vidya Academy of Science \& Technology \\ Thalakkottukara, Thrissur-680501, India
}

\author{
K. A. Germina \\ PG \& Research Department of Mathematics \\ Mary Matha Arts \& Science College \\ Mananthavady Wayanad-670645, India
}

\author{
K. P. Chithra \\ Naduvath Mana, Nandikkara \\ Thrissur-680301, India
}

\begin{abstract}
Let $\mathbb{N}_{0}$ be the set of all non-negative integers, let $X \subset \mathbb{N}_{0}$ and $\mathcal{P}(X)$ be the the power set of $X$. An integer additive set-labeling (IASL) of a graph $G$ is an injective function $f: V(G) \rightarrow \mathcal{P}\left(\mathbb{N}_{0}\right)$ such that the induced function $f^{+}: E(G) \rightarrow \mathcal{P}\left(\mathbb{N}_{0}\right)$ is defined by $f^{+}(u v)=f(u)+f(v)$, where $f(u)+f(v)$ is the sum set of $f(u)$ and $f(v)$. An IASL $f$ is said to be an integer additive set-indexer (IASI) of a graph $G$ if the induced edge function $f^{+}$is also injective. An integer additive set-labeling $f$ is said to be a weak integer additive set-labeling (WIASL) if $\left|f^{+}(u v)\right|=$ $\max (|f(u)|,|f(v)|) \forall u v \in E(G)$. The minimum cardinality of the ground set $X$ required for a given graph $G$ to admit an IASL is called the set-labeling number of the graph. In this paper, the notion of the weak set-labeling number of a graph $G$ is introduced as the minimum cardinality of $X$ so that $G$ admits a WIASL with respect to the ground set $X$ and the weak set-labeling numbers of certain graphs are discussed.
\end{abstract}

\section{Keywords:}

Integer additive set-labeled graphs; weak integer additive setlabeled graphs; weak set-labeling number of a graph.

\section{AMS Subject Classification: $05 \mathrm{C78}$}

\section{INTRODUCTION}

For all terms and definitions, not defined specifically in this paper, refer to [1], [6] and [14] and for different graph classes, further refer to [2] and [3]. Unless mentioned otherwise, all graphs considered here are simple, finite and have no isolated vertices.

The sum set of two sets $A$ and $B$, denoted $A+B$, is the set defined by $A+B=\{a+b: a \in A, b \in B\}$. If either $A$ or $B$ is countably infinite, then their sum set will also be countably infinite. Hence, all sets that are considered in this study are finite sets. The cardinality of a set $A$ is denoted by $|A|$. The power set of a set $A$ is denoted by $\mathcal{P}(A)$.

Using the concepts of sumsets, the notion of an integer additive set-labeling of a graph $G$ is introduced as follows.

Let $\mathbb{N}_{0}$ denote the set of all non-negative integers and $X$ be a subset of $\mathbb{N}_{0}$. An integer additive set-labeling (IASL, in short) of a graph $G$ is defined as an injective function $f: V(G) \rightarrow \mathcal{P}(X)$ such that the induced function $f^{+}: E(G) \rightarrow \mathcal{P}(X)$ is defined by $f^{+}(u v)=$ $f(u)+f(v)$, where $f(u)+f(v)$ is the sumset of the set-labels $f(u)$ and $f(v)$. A graph which admits an IASL is called an integer additive set-labeled graph (IASL-graph).

An integer additive set-indexer of a graph $G$ is an integer additive set-labeling $f: V(G) \rightarrow \mathcal{P}(X)$ such that the induced edge function $f^{+}: E(G) \rightarrow \mathcal{P}(X)$ is also injective.

Figure 1 depicts an integer additive set-labeling defined on a given graph $G$.

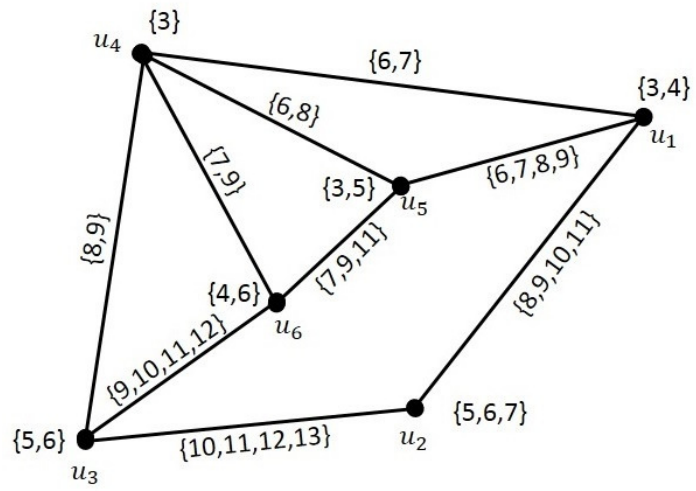

Fig. 1. An illustration to an IASL-graph.

The set-labeling number of a given graph $G$ is the minimum cardinality of the ground set $X$ so that the function $f: V(G) \rightarrow \mathcal{P}(X)$ is a WIASL of $G$. The set-labeling number of a graph $G$ is denoted by $\sigma(G)$.

DEFINITION 1.1. [8] A weak integer additive set-labeling $f$ of a graph $G$ is an IASL such that $\left|f^{+}(u v)\right|=\max (|f(u)|,|f(v)|)$ for all $u, v \in V(G)$. A weak IASI $f$ is said to be weakly uniform IASI if $\left|f^{+}(u v)\right|=k$, for all $u, v \in V(G)$ and for some positive integer $k$. A graph which admits a weak IASI may be called a weak integer additive set-labeled graph (WIASL-graph).

A WIASL $f$ of a given graph $G$ is said to be a weakly $k$-uniform IASL ( $k$-uniform WIASL) of $G$ if the set-labels of all edges of $G$ 
have the same cardinality $k$, where $k$ is a positive integer. If $G$ admits a WIASL, then it can be noted that the vertex set of $G$ can be partitioned into two sets such that the first set, say $V_{1}$, consists of all those vertices of $G$ having singleton set-labels and the other set, say $V_{2}$ consists of all those vertices having non-singleton set-labels. As a result, the following theorem have been established.

THEOREM 1.2. [5] A graph $G$ admits a k-uniform WIASL if and only if $G$ is bipartite or $k=1$.

The following result is a necessary and sufficient condition for a given graph to admit a weak integer additive set-labeling.

LEMMA 1.3. [8] A graph $G$ admits a weak integer additive setindexer if and only if every edge of $G$ has at least one mono-indexed end vertex.

In view of the above lemma, no two of its adjacent vertices can have non-singleton set-labels. Then, some of the adjacent vertices of $G$ can have singleton sets as their end vertices. In these cases, the set-label of the corresponding edges are also singleton sets. As a result, one can have the following theorem.

THEOREM 1.4. [8] A graph $G$ admits a WIASL if and only if $G$ is bipartite or it has some edges having singleton set-label.

In view of the above results, one can re-define a WIASL $f$ of a given graph $G$ as an IASL with respect to which the cardinality of the set-label of every edge of $G$ is equal to the cardinality of the set-label of at least one of its end vertices.

The following result is another important observation on WIASLgraphs.

THEOREM 1.5. [10] Let G be a WIASL-graph. Then, the minimum number of vertices of $G$ having singleton set-labels is equal to the vertex covering number $\alpha$ of $G$ and the maximum number of vertices of $G$ having non-singleton set-labels is equal to the independence number $\beta$ of $G$.

Figure2 illustrates a WIASL defined on a given graph $G$.

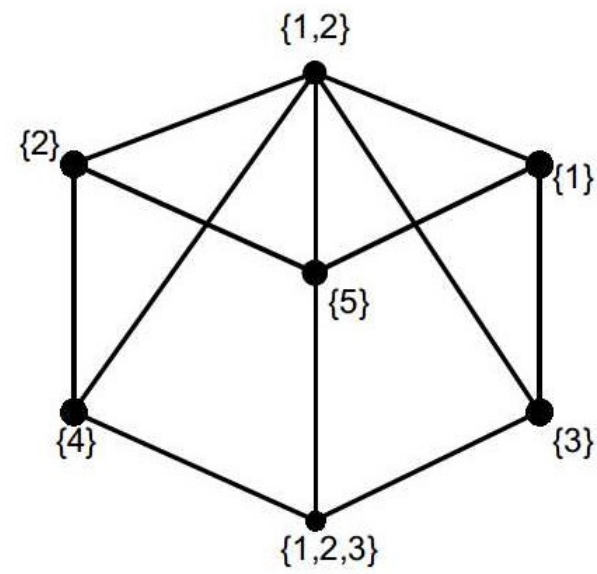

Fig. 2. An example to a WIASL-Graph

\section{WEAK SET-LABELING NUMBER OF GRAPHS}

Analogous to the terminology of set-labeling number of graphs which admit integer additive set-labelings, the notion of weak setlabeling number of a given graph $G$ is introduced as follows.

Definition 2.1. Let a function $f: V(G) \rightarrow \mathcal{P}(X)$ be an IASL of a given graph $G$, where $X$ is a non-empty finite ground set of non-negative integers. Then, the weak set-labeling number of a graph $G$ is the minimum cardinality of the ground set $X$, such that $f$ is a WIASL of $G$. The weak set-labeling number of a graph $G$ is denoted by $\sigma^{*}(G)$.

No IASL-graphs considered in the following discussion are 1uniform, unless specified otherwise.

In the following discussion, the weak set-labeling numbers of different standard graphs are determined. Let us begin with a path graph $P_{n}$ on $n$ vertices.

THEOREM 2.2. The weak set-labeling number of a path $P_{n}$ is $2+\left\lfloor\frac{n}{2}\right\rfloor$.

PROOF. Let $\left\{v_{i}, v_{2}, v_{3}, \ldots, v_{n}\right\}$ be the vertex set of the graph $P_{n}$. Note that a set-labeling in such a way that no two adjacent vertices in $P_{n}$ can have non-singleton set-labels is required. Hence, the vertices of $P_{n}$ are to labeled alternately by singleton and nonsingleton sets of non-negative integers. In this context, the following cases are to be considered.

Case-1: Let $n$ be even. Then, $n=2 r$ for some positive integer $r$. Label the vertices of $P_{n}$ by singleton sets and 2-element sets of non-negative integers as follows. Let $f\left(v_{2}\right)=\{1\}, f\left(v_{4}\right)=$ $\{2\}, f\left(v_{6}\right)=\{3\}, \ldots, f\left(v_{2 r}\right)=\{r\}$. Now, label odd vertices in reverse order as follows. Let $f\left(v_{2 r-1}\right)=\{1,2\}, f\left(v_{2 r-3}\right)=$ $\{2,3\}, f\left(v_{2 r-5}\right)=\{3,4\}, \ldots, v_{3}=\{r-1, r\}, f\left(v_{1}\right)=\{r, 1\}$. Then, the set-labels of the edges of $G$ are $v_{n} v_{n-1}=\{r+1, r+2\}$ $v_{n-1} v_{n-2}=\{1+r-1,2+r-1\}=\{r+1, r+2\}$ and so on. The set-labels of all edges other than the edge $v_{1} v_{2}$ are either $\{\mathrm{r}+1, \mathrm{r}+2\} \operatorname{or}\{r, r+1\}$ and the set-label of $v_{1} v_{2}$ is $\{2, r+1\}$. That is, $f^{+}(E)=\{\{2, r+1\},\{r, r+1\},\{r+1, r+2\}\}$. Now, choose $X=f(V) \cup f^{+}(E)=\{1,2,3, \ldots, r+1, r+2\}$. Clearly, $f$ : $V(G) \rightarrow \mathcal{P}(X)$ is a WIASL defined on $P_{n}$ and hence, $\sigma^{*}\left(P_{n}\right)=$ $2+\frac{n}{2}$.

Case-2: Let $n$ be odd. Then, $n=2 r+1$, for some positive integer $r$. Label the vertices of $P_{n}$ as follows. Let $f\left(v_{2}\right)=\{1\}, f\left(v_{4}\right)=$ $\{2\}, f\left(v_{6}\right)=\{3\}, \ldots, f\left(v_{2 r}\right)=\{r\}$. Now, label the remaining vertices in the reverse order as follows. Let $f\left(v_{2 r+1}\right)=$ $\{1,2\}, f\left(v_{2 r-1}\right)=\{2,3\}, f\left(v_{2 r-3}\right)=\{3,4\}, \ldots, v_{5}=\{r-$ $1, r\}, f\left(v_{3}\right)=\{r, 1\}$ and $f\left(v_{1}\right)=\{1,2,3\}$. Therefore, as in the previous case, $f^{+}(E)=\{\{2,3,4\},\{r, r+1\},\{r+1, r+2\}\}$. Then, $X=f(V) \cup f^{+}(E)=\{1,2,3, \ldots, r+1, r+2\}$. Therefore, the weak set-labeling number of $P_{n}$ is $r+2=2+\frac{n-1}{2}$.

Combining the above two cases, $\sigma^{*}\left(P_{n}\right)=2+\left\lfloor\frac{n}{2}\right\rfloor$.

Next, the weak set-labeling number of cycle graphs is determined in the following way.

THEOREM 2.3. The weak set-labeling number of a cycle $C_{n}$ is $2+\left\lfloor\frac{n}{2}\right\rfloor$.

Proof. Let $C_{n}: v_{i} v_{2} v_{3} \ldots v_{n} v_{1}$ be a cycle on $n$ vertices. We label the vertices alternately by singleton and non-singleton sets of non-negative integers. Here, the following cases are to be considered.

Case-1: Let $n$ be even. Then, $n=2 r$ for some positive integer $r$. Label the vertices of $C_{n}$ by singleton sets and 2-element sets of 
non-negative integers as follows. Let $f\left(v_{2 i}\right)=\{i\}$ for all $1 \leq i \leq$ $\frac{n}{2}$. Label the odd vertices such that $f\left(v_{2 r-1}\right)=\{1,2\}, f\left(v_{2 r-3}\right)=$ $\{2,3\}, f\left(v_{2 r-5}\right)=\{3,4\}, \ldots, v_{3}=\{r-1, r\}, f\left(v_{1}\right)=\{r, 1\}$. Then, all edges of $C_{n}$ except $v_{1} v_{2}$ and $v_{2} v_{3}$ have the set-labels either $\{r, r+1\}$ or $\{r+1, r+2\}$. Also, $f\left(v_{1} v_{2}\right)=\{2, r+1\}$ and $f\left(v_{2} v_{3}\right)=\{3, r+2\}$. Therefore, Choose $X=\{1,2,3, \ldots, r+$ $1, r+2\}$. Hence, in this case $\sigma^{*}\left(C_{n}\right)=r+2=2+\frac{n}{2}$.

Case-2: Let $n$ be odd. Then, $n=2 r+1$, for some positive integer $r$. For an WIASL-graph $C_{n}$, there exist $r+1$ singleton set labels and $r$ non-singleton sets. Label the vertices of $C_{n}$ as follows. Now, let $f\left(v_{1}\right)=\{1\}, f\left(v_{3}\right)=\{2\}, f\left(v_{5}\right)=\{3\}, \ldots, f\left(v_{2 r+1}\right)=$ $\{r+1\}$ and let $f\left(v_{2}\right)=\{r, r-1\}, f\left(v_{4}\right)=\{r-1, r-2\}, f\left(v_{6}\right)=$ $\{r-2, r-3\}, \ldots f\left(v_{2 r}\right)=\{1,2\}$. Then, all edges other than the edge $v_{n} v_{1}$ have the set-label either $\{r, r+1\}$ or $\{r+1, r+2\}$ and the edge $v_{n} v_{1}$ has the set-label $\{r+2\}$. Therefore, the set $X$ can be chosen as the set $f(V) \cup f^{+}(E)=\{1,2,3, \ldots, r+1, r+2\}$ and hence $\sigma^{*}(G)=r+2=2+\frac{n-1}{2}$.

Combining the above two cases, one can note that $\sigma^{*}\left(C_{n}\right)=2+$ $\left\lfloor\frac{n}{2}\right\rfloor$.

The weak set-labeling number of a complete graph is determined in the following theorem.

THEOREM 2.4. The weak set-labeling number of a complete graph $K_{n}$ is $2 n-3$.

Proof. . Let $V\left(K_{n}\right)=\left\{v_{1}, v_{2}, v_{3}, \ldots, v_{n}\right\}$. Since all vertex of $K_{n}$ are mutually adjacent, at most one vertex can have a nonsingleton set-label. Let $f$ be an IASL defined on $K_{n}$ which assigns the set-labels to the vertices of $K_{n}$ as follows. Let $f\left(v_{i}\right)=\{i\}$ for $1 \leq i \leq n-1$ and let $f\left(v_{n}\right)=\{1,2\}$. Then, $f^{+}\left(E\left(K_{n}\right)\right)=$ $\{3,4,5, \ldots, 2 n-3\}$. That is, the ground set with minimum cardinality is the set $f(V) \cup f^{+}(E)=\{1,2,3, \ldots, 2 n-3\}$. Hence, $\sigma^{*}(G)=2 n-3$.

Figure 3 depicts a complete graph on 6 vertices and weak setlabeling number 9 .

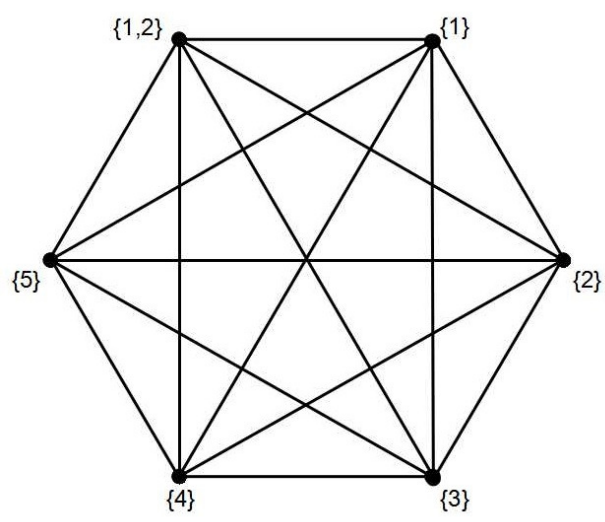

Fig. 3. A complete graph with weak set-labeling number 9 .

\section{WEAK SET-LABELING NUMBER OF CERTAIN GRAPH CLASSES}

Let us now proceed to discuss the weak set-labeling number of certain graphs that are generated from cycles. A graph that needs to be considered first among these types of graphs is a wheel graph $W_{n+1}$ which is a graph obtained by drawing edges from all vertices of a cycle to an external vertex (see [3]). That is, $W_{n+1}=C_{n}+K_{1}$. The following theorem establishes the weak set-labeling number of a wheel graph.

THEOREM 3.1. The weak set-labeling number of a wheel graph $W_{n+1}=C_{n}+K_{1}$ is $3+\left\lfloor\frac{n}{2}\right\rfloor$.

Proof. Let $G=C_{n}+K_{1}$, where $C_{n}: v_{1} v_{2} v_{3} \ldots v_{n} v_{1}$ and $K_{1}=\{v\}$. Label the vertices of $C_{n}$ in $G$ as explained in Theorem 2.3 What remains is to label the vertex $v$. Since all sets of the form $\{i, i+1\}$ have already been used for labeling the vertices of $C_{n}$, label $v$ by the set $\{1,3\}$. It can be observed that the only element in a set-label of the edge $v v_{i}$ of $G$, which is not in any set-label of the elements of $C_{n}$ is $r+3$, where $r=\left\lfloor\frac{n}{2}\right\rfloor$. Hence, $f(V(G)) \cup f^{+}(E(G))=\{1,2,3, \ldots, r+3\}$. Therefore, the weak set-labeling number of the wheel graph $G$ is $3+\left\lfloor\frac{n}{2}\right\rfloor$.

Figure 4 illustrates the wheel graph $W_{7}$ with weak set-labeling number 6 .

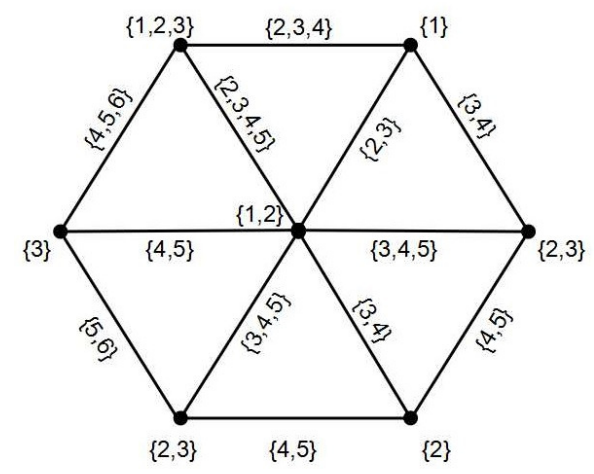

Fig. 4. A wheel graph with weak set-labeling number 6 .

A helm graph, (see [3]) denoted by $H_{n}$, is a graph obtained by attaching a pendant edge to each vertex of the outer cycle of a wheel graph $W_{n+1}$. Then, the helm graph $H_{n}$ has $2 n+1$ vertices and $3 n$ edges. That is, $H_{n}=W_{n+1} \odot K_{1}$, where $\odot$ is the corona of two graphs. The following theorem establishes the weak set-labeling number of the helm graph $H_{n}$.

THEOREM 3.2. The weak set-labeling number of a helm graph $H_{n}$ is $n+3$.

Proof. Let $G$ be a helm graph on $2 n+1$ vertices. Then, $G=$ $\left(C_{n}+K_{1}\right) \odot K_{1}$. Let $\{v\}$ be the central vertex, $\left\{v_{1}, v_{2}, v_{3}, \ldots, v_{n}\right\}$ be the vertices in the cycle $C_{n}$ of $G$ and let $\left\{u_{1}, u_{2}, u_{3}, \ldots, u_{n}\right\}$ be the pendant vertices of $G$ such that the vertex $u_{i}$ is adjacent to the vertex $v_{i}, 1 \leq i \leq n$. Since a vertex cover of $H_{n}$ contains $n$ elements, by Theorem 1.5 with respect to any WIASL defined on $G$, there must be at least $n$ vertices in $G$ must have singleton set-labels. Now, define an IASL $f$ on $G$ as follows. Label the vertices of $C_{n}$ by $f\left(v_{i}\right)=\{i\}$, where $1 \leq i \leq n$. Then, label the pendant vertices of $G$ such that $f\left(u_{n}\right)=\{1,2\}, f\left(u_{n-1}\right)=\{2,3\}, f\left(u_{n-2}\right)=$ $\{3,4\}, \ldots, f\left(u_{2}\right)=\{n-1, n\}, f\left(u_{1}\right)=\{1, n\}$. The only vertex that remains to be labeled is the central vertex $v$. Since all subsets of $X$ of the form $\{i, i+1\}$ have been used for labeling other vertices, choose the set $\{1,3\}$ to label the vertex $v$. Then, 
$f(V(G)) \cup f^{+}(E(G))=\{1,2,3, \ldots, n+3\}$. Since the minimal ground set $X$ is $f(V(G)) \cup f^{+}(E(G))$, then $\sigma^{*}(G)=n+3$.

Figure 4 illustrates the helm graph on 13 vertices with weak setlabeling number 7 .

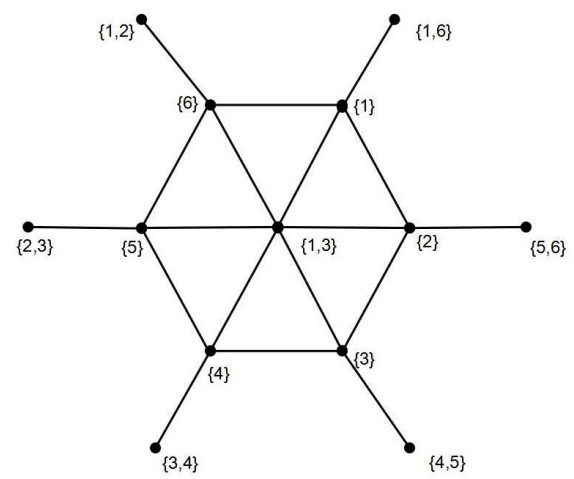

Fig. 5. A helm graph with weak set-labeling number 9 .

A friendship graph $F_{n}$ is a graph obtained by identifying one end vertex of $n$ triangles. A friendship graph has $2 n+1$ vertices and $3 n$ edges. The following theorem determines the weak set-labeling number of a friendship graph.

THEOREM 3.3. The weak set-labeling number of a friendship graph $F_{n}$ is $n+3$.

Proof. Let $v$ be the central vertex of the friendship graph $F_{n}$. Divide the remaining vertices of $F_{n}$ in to two sets $U=$ $\left\{u_{1} u_{2}, u_{3}, \ldots, u_{n}\right\}$ and $W=\left\{w_{1}, w_{2}, w_{3}, \ldots, w_{n}\right\}$ such that the graph induced by the vertices $v, u_{i}, w_{i}$ is a triangle in $F_{n}$, where $1 \leq i \leq n$. Let $f$ be an IASL defined on $F_{n}$ with respect to which, the set-labeling of the vertices of $F_{n}$ is done in the following way. Let $f(v)=\{1\}$ and $f\left(u_{i}\right)=\{i+1\}$, where $1 \leq i \leq n$. Now, label the set $W$ in such a way that $f\left(w_{n}\right)=\{1,2\}, f\left(w_{n-1}\right)=$ $\{2,3\}, \ldots, f\left(w_{2}\right)=\{n-1, n\}, f\left(w_{1}\right)=\{n, n+1\}$. Then, $f^{+}(E)=\{2,3,4, \ldots, n+3\}$. Hence, the minimal ground set is $f(V) \cup f^{+}(E)=\{1,2,3, \ldots, n+3\}$ and $\sigma^{*}(G)=n+3$.

Figure 6 illustrates a friendship graph $F_{4}$ with weak set-labeling number 7 .

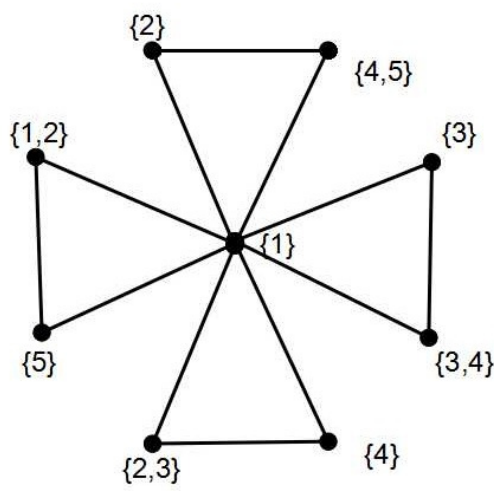

Fig. 6. A friendship graph with weak set-labeling number 7 .
A sunlet graph $S L_{n}$ is a graph obtained by attaching a pendant edge to all vertices of a cycle $C_{n}$ (see [13]). The sunlet graph $S L_{n}$ has $2 n$ vertices and $2 n$ edges. The following theorem establishes the weak set-labeling number of a sunlet graph.

THEOREM 3.4. The weak set-labeling number of a sunlet graph $S L_{n}=C_{n} \odot K_{1}$ is $n+2$.

Proof. We have $G=C_{n} \odot K_{1}$. Let $\left\{v_{1}, v_{2}, v_{3}, \ldots, v_{n}\right\}$ be the vertices of $C_{n}$ and $\left\{u_{1}, u_{2}, u_{3} \ldots, u_{n}\right\}$ be the pendant vertices of $G$. Let $f$ be be an IASL defined on $S L_{n}$ which assigns set-labels to the vertices of $S L_{n}$ as follows. Let $f\left(u_{n}\right)=\{1,2\}, f\left(u_{n-1}\right)=$ $\{2,3\}, f\left(u_{n-2}\right)=\{3,4\}, \ldots, f\left(u_{2}\right)=\{n-1, n\}, f\left(v_{1}\right)=$ $\{1, n\}$. Then, as explained in previous theorems, the minimal ground set $X=f(V(G)) \cup f^{+}(E(G))=\{1,2,3, \ldots, n+2\}$. Hence, for the sunlet graph $G=C_{n} \odot K_{1}, \sigma^{*}(G)=n+2$.

Another similar graph, that is considered here, is a sun graph which is defined as follows. A sun graph $S_{n}$ is a graph obtained by replacing every edge of a cycle $C_{n}$ by a triangle $C_{3}$ (see |2|). A sun graph also has $2 n$ vertices. The same set-labeling, as explained in the previous theorem, can be applied to the vertices of a sun graph $S_{n}$ and hence one can establish the following theorem.

THEOREM 3.5. The weak set-labeling number of a sun graph $S_{n}$ is $n+3$

Proof. Let $\left\{v_{1}, v_{2}, v_{3}, \ldots, v_{n}\right\}$ be the vertex set of the cycle $C_{n}$ and let $\left\{u_{1}, u_{2}, u_{3}, \ldots, u_{n}\right\}$ be the independent vertices of $S_{n}$, such that the vertex $u_{i}$ is adjacent to the vertices $v_{i}$ and $v_{i+1}$, in the sense that $v_{n+1}=v_{1}$. As explained in the previous theorem, label the vertices of $C_{n}$ as $f\left(v_{i}\right)=\{i\}$ and label the independent vertices such that $f\left(u_{n}\right)=\{1,2\}, f\left(u_{n-1}\right)=$ $\{2,3\}, f\left(u_{n-2}\right)=\{3,4\}, \ldots, f\left(u_{2}\right)=\{n-1, n\}, f\left(v_{1}\right)=$ $\{1, n\}$. Then, $f^{+}\left(E\left(S_{n}\right)\right)=\{2,3,4, \ldots, n+3\}$. Hence, the minimal ground set $X=f(V) \cup f^{+}(E)=\{1,2,3, \ldots, n+3\}$. Therefore, the weak set-labeling number of the graph $S_{n}$ is $n+3$.

Figure 7 illustrates Theorem 3.5

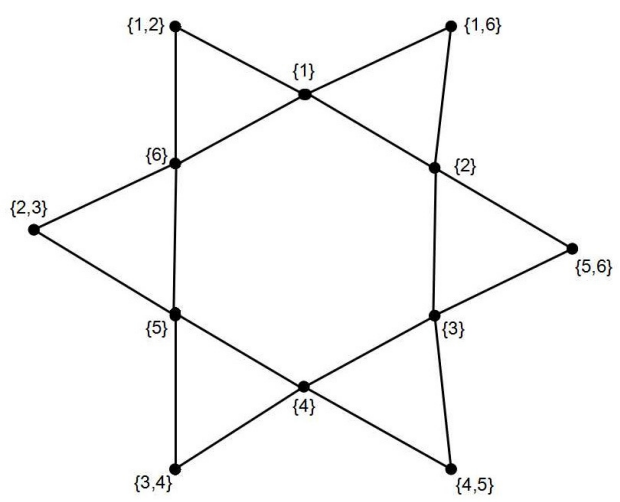

Fig. 7. A sun graph with weak set-labeling number 6

The next graph to study next for weak set-labeling number is a complete sun graph which is defined as follows. A sun (or trampoline) (see [2]) is a $G$ on $n$ vertices for some $n>3$ whose vertex set can be partitioned into two sets, $W=\left\{w_{1}, w_{2}, w_{3}, \ldots, w_{n}\right\}$, $U=\left\{u_{1}, u_{2}, u_{3}, \ldots, u_{n}\right\}$, such that $W$ is independent and for each $i$ and $j$, the vertex $w_{i}$ is adjacent to the vertices $v_{i}$ and $v_{i+1}$, in the sense that $v_{n+1}=v_{1}$. A complete sun is a sun $G$ in which the 
induced subgraph $\langle U\rangle$ is a complete graph. The following theorem determines the weak set-labeling number of a complete sun graph. A sun (or complete sun) has $2 n$ vertices.

THEOREM 3.6. The weak set-labeling number of a complete sun graph on $2 n$ vertices is $n+3$.

Proof. Let $G$ be a complete graph on $2 n$ vertices whose vertex sets are partitioned in to two sets $U$ and $W$, where the induced subgraph $\langle U\rangle$ of $G$ is a complete graph and $W$ is an independent set. As usual, label the vertices of $C_{n}$ as $f\left(u_{i}\right)=\{i\}$ and label the vertices in $W$ in such a way that $f\left(w_{n}\right)=\{1,2\}, f\left(w_{n-1}\right)=$ $\{2,3\}, f\left(w_{n-2}\right)=\{3,4\}, \ldots, f\left(w_{2}\right)=\{n-1, n\}, f\left(w_{1}\right)=$ $\{1, n\}$. Then, $f^{+}(E)=\{2,3,4, \ldots, n+3\}$ and $f(V) \cup f^{+}(E)=$ $\{1,2,3, \ldots, n+3\}$. Hence, the minimum required cardinality for the ground set $X$ is $\sigma^{*}(G)=\left|f(V) \cup f^{+}(E)\right|=n+3$.

Figure 8 illustrates a complete sun graph with a weak set-labeling number 7 .

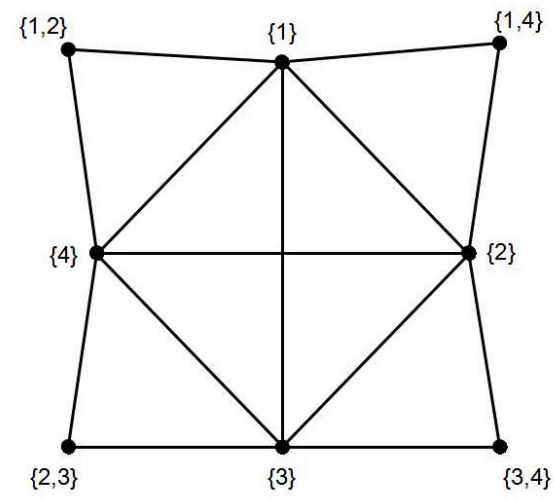

Fig. 8. A complete sun graph with weak set-labeling number 7

\section{SCOPE FOR FURTHER STUDIES}

In this paper, the discussion was on the weak set-labeling number and weak set-indexing number of certain classes of graphs. Certain Problems in this area are still open. Some of the open problems that identified in this area are the following.

PROBLEM 4.1. Determine the weak set-labeling number of a bipartite graphs, both regular and biregular.

Problem 4.2. Determine the weak set-labeling number of certain graphs like gear graphs, lobsters, double wheel graphs etc.

PROBLEM 4.3. Determine the weak set-labeling number of certain graphs like double helm graphs, web graphs, windmill graphs etc.

Problem 4.4. Determine the weak set-labeling number of split graphs, complete split graphs, bisplit graphs etc.

Problem 4.5. Determine the weak set-labeling number of arbitrary sun graphs, rising sun graphs and partial sun graphs etc.

PROBLEM 4.6. Determine the weak set-labeling number of graphs, which admit a 1-uniform IASL.
Analogous to the weak set-labeling number of graphs, the notion of the weak set-indexing number of a graph $G$ can be defined as follows.

DEFINITION 4.7. The minimum cardinality of the ground set $X$, so that the function $f: V(G) \rightarrow \mathcal{P}(X)$ is a WIASI of $G$, is called the weak set-indexing number of $G$ and is denoted by $\sigma^{\#}(G)$.

Determining the weak set-indexing number of different graph classes is also an open problem.

There are several other types of standard graphs and named graphs whose weak set-labeling numbers and weak-set-indexing numbers can be calculated. Determining these parameters of different graph operations and graph parameters are also promising. Similar parameters corresponding to other types of integer additive set-labelings such as strong integer additive set-labelings, arithmetic integer additive set-labelings, exquisite integer additive setlabelings etc. are also worthy for future studies. All these facts show that there are wide scope for further studies in this direction.

\section{REFERENCES}

[1] J. A. Bondy and U. S. R. Murty, Graph Theory, Springer, 2008.

[2] A. Brandstädt, V. B. Le and J. P. Spinrad, Graph Classes: A Survey, SIAM, Philadelphia, 1999.

[3] J. A. Gallian, A Dynamic Survey of Graph Labelling, The Electronic Journal of Combinatorics, DS \#16, 2011.

[4] K. A. Germina and T. M. K. Anandavally, Integer Additive Set-Indexers of a Graph: Sum Square Graphs, Journal of Combinatorics, Information and System Sciences, 37(24)(2012), 345-358.

[5] K. A. Germina and N. K. Sudev, On Weakly Uniform Integer Additive Set-Indexers of Graphs, International Mathematical Forum, 8(37)(2013), 1827-1834. DOI:10.12988/imf.2013.310188

[6] F. Harary, Graph Theory, Addison-Wesley Publishing Company Inc., 1994.

[7] N. K. Sudev and K. A. Germina, On Integer Additive SetIndexers of Graphs, International Journal of Mathematical Sciences \& Engineering Applications, 8(II)(2014), 11-22.

[8] N. K. Sudev and K. A. Germina, A Characterisation of Weak Integer Additive Set-Indexers of Graphs, ISPACS Journal of Fuzzy Set Valued Analysis, 2014(2014), 7 pages, DOI: 10.5899/2014/jfsva-00189.

[9] N. K. Sudev and K. A. Germina, Weak Integer Additive SetIndexers of Certain Graph Operations, Global Journal of Mathematical Sciences: Theory \& Practical, 6(1)(2014), 2536.

[10] N. K. Sudev and K. A. Germina, A Note on Sparing Number of Graphs, Advances and Applications in Discrete Mathematics, 14(1)(2014),50-65.

[11] N. K. Sudev and K. A. Germina, Weak Integer Additive SetIndexers of Certain Graph Classes, to appear in Journal of Discrete Mathematical Sciences \& Cryptography.

[12] N. K. Sudev, K. A. Germina and K. P. Chithra, Weak Integer Additive Set-Labeled Graphs: A Creative Review, to appear in Asian European Journal of Mathematics.

[13] W. D. Wallis, Beginner's Guide to Graph Theory, Birkhäuser, Boston, 2007. 
[14] D. B. West, Introduction to Graph Theory, Pearson Education Inc., 2001.

[15] Information System on Graph Classes and their Inclusions, http://www.graphclasses.org 